\title{
Desarrollo Embrionario del Capaz Pimelodus grosskopfii (Steindachner, 1879)
}

\author{
Embryonic Development of Capaz Pimelodus grosskopfii (Steindachner, 1879)
}

"Rubén D. Valbuena-Villarreal; **Beatriz E. Zapata-Berruecos; "***Carlos David-Ruales \& **Pablo E. Cruz-Casallas

VALBUENA-VILLARREAL, R. D.; ZAPATA-BERRUECOS B. E.; DAVID-RUALES, C. \& CRUZ-CASALLAS, P. E. Desarrollo embrionario del capaz Pimelodus grosskopfii (Steindachner, 1879). Int. J. Morphol., 30(1):150-156, 2012.

RESUMEN: El conocimiento del desarrollo embrionario en los peces es especialmente importante en especies nativas con potencial para la piscicultura, en virtud que permite identificar eventos morfológicos y cronológicos, necesarios para establecer prácticas de manejo durante las fases de incubación y larvicultura. El capaz (Pimelodus grosskopfii) es una especie con potencial para cultivo comercial, por sus hábitos alimenticios omnívoros y aceptación de su carne en el mercado. Para estudiar el desarrollo embrionario de la especie, ejemplares adultos sexualmente maduros fueron inducidos a la reproducción con extracto de hipófisis de carpa (5,75 y 4,0 mg. $\mathrm{Kg}^{-1}$, hembras y machos, respectivamente). Los óvulos seminados fueron incubados en un sistema de flujo ascendente de $30 \mathrm{~L}$ a $27 \pm 1$ ${ }^{\circ} \mathrm{C}$. Las muestras $(\mathrm{n}=30)$ fueron colectadas al momento de la extrusión, durante la fertilización y cada 15 minutos a partir de las 0 horas postfertilización (HPF) hasta las 2 horas y cada 30 minutos desde las 2 HPF hasta 5 HPF; finalmente, entre las 5 HPF y la eclosión, cada 60 minutos. Los óvulos fertilizados presentaron forma esférica, sin adherencias y con amplio espacio perivitelino. El desarrollo embrionario finalizó a las $12 \mathrm{HPF}$. La diferenciación del polo animal y vegetal ocurrió a las 0,2 HPF, el primer clivaje a las 0,3 HPF, el blastodisco alto y estratificado a las 1,8 HPF, el blastodisco achatado a las 3,3 HPF, la epibolia < a 50 \% se observó a las 4 HPF, el cierre del blastoporo a las 5,7 HPF, la diferenciación cráneo caudal e inicio de la neurolación a las 7 HPF, la diferenciación de las vesículas ópticas, óticas y vesícula de Kupffer a las 8,5 HPF, la liberación de la cola del vitelo a las 10 HPF, los primeros movimientos se observaron a las 10,5 HPF y finalmente la eclosión ocurrió a las 12 HPF. Las larvas al eclosionar presentaron una longitud total de $2987 \pm 67 \mu \mathrm{m}$, sin pigmentación, tracto digestivo rudimentario, sin abertura bucal ni anal y presencia de cromatóforos en el saco vitelino. grosskopfii.

PALABRAS CLAVE: Blastómero; Blastulación; Desarrollo embrionario; Gastrulación; Organogénesis; Pimelodus

\section{INTRODUCCIÓN}

El Capaz (Pimelodus grosskopfii) es una especie de la familia Pimelodidae, que en Colombia habita las cuencas de los ríos Magdalena, Cauca, San Jorge, Sinú, Cesar, Atrato, Baudó y Catatumbo (Mojica, 1999; Ortega-Lara et al., 2000; Villa-Navarro, 2002); también ha sido reportado en el embalse de Betania (Huila) (Villa-Navarro, 2002). Es una especie omnívora, con preferencia por insectos, macroinvertebrados y peces (Villa-Navarro, 1999).

La investigación con silúridos en Colombia, especialmente de la familia Pimelodidae, se ha limitado a la ejecución de trabajos sobre aspectos de su biología básica y al desarrollo de protocolos para su reproducción inducida bajo condiciones de cautiverio; pero, hasta el presente, ninguna de las especies de este grupo dispone de un paquete tecnológico para su cultivo a escala comercial, encontrándose aún grandes vacíos especialmente para el manejo de su incubación y larvicultura. Por esta razón, la carne de silúridos que se consume actualmente en el país proviene exclusivamente de la actividad pesquera, pero debido a la sobrepesca y a la utilización de métodos y medios de captura inadecuados, esta actividad ha contribuido al deterioro de los hábitats de estas especies, causando una dramática disminución de las poblaciones naturales y por consiguiente de los volúmenes de captura (CCI, 2006). El capaz (P. grosskopfii), por las características de calidad de su carne (blanca y ausencia de espinas intramusculares) tiene alta aceptación en el mercado nacional y un significativo valor comercial; lo cual,

\footnotetext{
Facultad de Ciencias Exactas y Naturales, Universidad Surcolombiana, Neiva, Colombia.

** Grupo de Investigación sobre Reproducción y Toxicología de Organismos Acuáticos - GRITOX. Instituto de Acuicultura, Facultad de Ciencias Agropecuarias y Recursos Naturales, Universidad de los Llanos, Villavicencio, Colombia. E-mail: pecruzcasallas@unillanos.edu.co

*** Grupo de investigación en Producción, Desarrollo y Transformación Agropecuaria, Corporación Universidad Lasallista, Medellín, Colombia.
} 
junto con su potencial reproductivo mostrado en ensayos preliminares bajo condiciones de cautiverio, hacen de esta una especie nativa promisoria para el desarrollo de tecnologías de cultivo y una alternativa para diversificar la piscicultura colombiana.

El estudio del desarrollo embrionario es útil para identificar cronológicamente los eventos morfológicos que llevan a la formación de un nuevo individuo, permitiendo establecer las técnicas más adecuadas para la incubación y la larvicultura de la especie. Estudios recientes en yaque (Leiarius marmoratus) (Zapata-Berruecos et al., 2008) y en bagre rayado (Psedoplastystoma sp.) (Zapata-Berruecos et al., 2007), muestran que entre especies de la misma familia existen marcadas diferencias en cuanto a la cronología del desarrollo embrionario, lo cual hace necesario realizar trabajos para conocer la embriología de cada especie en particular. En consecuencia, el objetivo del presente trabajo fue describir los principales eventos del desarrollo embrionario de capaz (P. grosskopfii), bajo condiciones controladas de incubación.

\section{MATERIAL Y MÉTODO}

Aval de Comité de ética. Este trabajo contó para su ejecución con los avales de los Comités de Ética para la experimentación con animales (CEEA) de la Universidad Surcolombiana y de la Universidad de los Llanos (Colombia).

Localización. El ensayo fue realizado en la Estación Piscícola "Piedra-Pintada" de la Central de Cooperativas de Caficultores del Huila (CENTRACAFE), ubicada en el Municipio de Aipe, Departamento del Huila (Colombia), localizada a una altura de $390 \mathrm{msnm}$, con temperatura promedio anual de $29,5^{\circ} \mathrm{C}$.

Reproducción inducida. Se seleccionaron hembras y machos capturados del ambiente, con peso corporal de $407 \pm$ 68 y $183 \pm 40 \mathrm{~g}$, respectivamente. Después de un periodo de adaptación en estanques en tierra de dos meses, los peces fueron inducidos a la maduración final de sus gónadas mediante la aplicación intramuscular de extracto de hipófisis de carpa (EHC), utilizando un protocolo establecido para la especie, el cual consiste en administrar $5,75 \mathrm{mg} \cdot \mathrm{Kg}^{-1}$ para las hembras, aplicados en tres inyecciones [0,25 $(0 \mathrm{~h}), 0,5$ (24 h) y $\left.5,0 \mathrm{mg} \cdot \mathrm{Kg}^{-1}(36 \mathrm{~h})\right]$ y para machos $4 \mathrm{mg} \cdot \mathrm{Kg}^{-1}$, en una sola inyección, administrada $34 \mathrm{~h}$ después de la primera inyección de las hembras.

Los óvulos fueron obtenidos por extrusión, inme- diatamente después la ovulación, la cual ocurrió aproximadamente $7 \mathrm{~h}$ después de la aplicación de la última inyección de EHC. El semen fue obtenido con la ayuda de una sonda introducida por el conducto espermático y ejerciendo presión sobre las paredes de la cavidad celómica. Inmediatamente después de su recolección, el semen obtenido fue mezclado con los óvulos y activado adicionando a la mezcla $20 \mathrm{~mL}$ de agua. Una vez ocurrida la hidratación, los óvulos seminados fueron transferidos a incubadoras tipo Woynarovich de $30 \mathrm{~L}$, conservando un flujo de agua de 2 $3 \mathrm{~L} \cdot \mathrm{min}^{-1}$.

Toma de muestras. Para determinar la duración del desarrollo embrionario, se consideró como momento cero la hora de activación de los gametos (fertilización) y como hora final el momento en que el $90 \%$ de los óvulos de una muestra aleatoria alcanzó la eclosión (Johnston et al., 1995). Durante la etapa de desarrollo embrionario fueron observadas 30 muestras, en cada uno de los momentos evaluados. Las muestras de embriones en desarrollo fueron fijadas con formol tamponado al $4 \%$. En cada una de las muestras se realizó identificación del estadio de desarrollo embrionario y se estableció el porcentaje relativo de cada uno dentro de cada momento de muestreo.

Las muestras fueron tomadas según la metodología de Novoa \& Cataño-Vergara (2005) así: cada 15 minutos a partir de la 0 hora postfertilización (HPF) hasta las 2 HPF; a partir de este momento, cada 30 minutos hasta las $5 \mathrm{HPF}$ y cada hora desde la 6 HPF hasta el momento de la eclosión. El material biológico fue colocado en cajas de Petri y los registros fotográficos fueron realizados por medio de un estereoscopio (Nikon, SMZ800), para su posterior análisis. Durante el proceso de incubación se monitoreó la temperatura, pH y oxígeno disuelto.

\section{RESULTADOS}

Calidad del Agua. El desarrollo embrionario ocurrió bajo las siguientes características físicas y químicas del agua: temperatura: $27 \pm 1,0^{\circ} \mathrm{C}$; Oxígeno disuelto: $6,0 \pm 1 \mathrm{mg} \cdot \mathrm{L}^{-1} ; \mathrm{y} \mathrm{pH}$ : $7,5 \pm 0,5$.

Descripción del óvulo. Los óvulos desovados, no hidratados, fueron esféricos, no adherentes, con diámetro promedio de $1200 \pm 16 \mu \mathrm{m}$, de color amarillo claro (Fig. 1A). Después de la hidratación, mostraron un amplio espacio perivitelino y un aumento significativo de su diámetro $(2700 \mu \mathrm{m})$. Se visualizó la presencia de una doble membrana, con un corion transparente, lo cual permitió la observación de los diferentes eventos ontogénicos (Fig. 1B). 
Blastomeración. En P. grosskopfii, la blastomeración es de tipo meroblástica, observándose la diferenciación entre el polo animal y vegetal a partir de las 0,20 HPF; los óvulos se mostraron telolecíticos, como se muestra en la Fig. 1C.

El polo animal presentó sucesivas divisiones mitóticas originando un blastodisco segmentado. El primer clivaje o segmentación discoidal ocurrió a las 0,3 HPF, di- vidiendo el blastodisco verticalmente en dos células simétricas. La segunda división se observó a las 0,45 HPF originándose 4 blastómeros (Fig. 1D); el tercer plano de división (0,55 HPF) fue doble y paralelo al primero, originando 8 blastómeros (Fig. 1E), el cuarto clivaje (1,0 HPF) originó 16 blastómeros (Fig. $1 \mathrm{~F}$ ). Con el aumento de las divisiones celulares se dificultó la visualización de los blastómeros y por lo tanto su conteo.
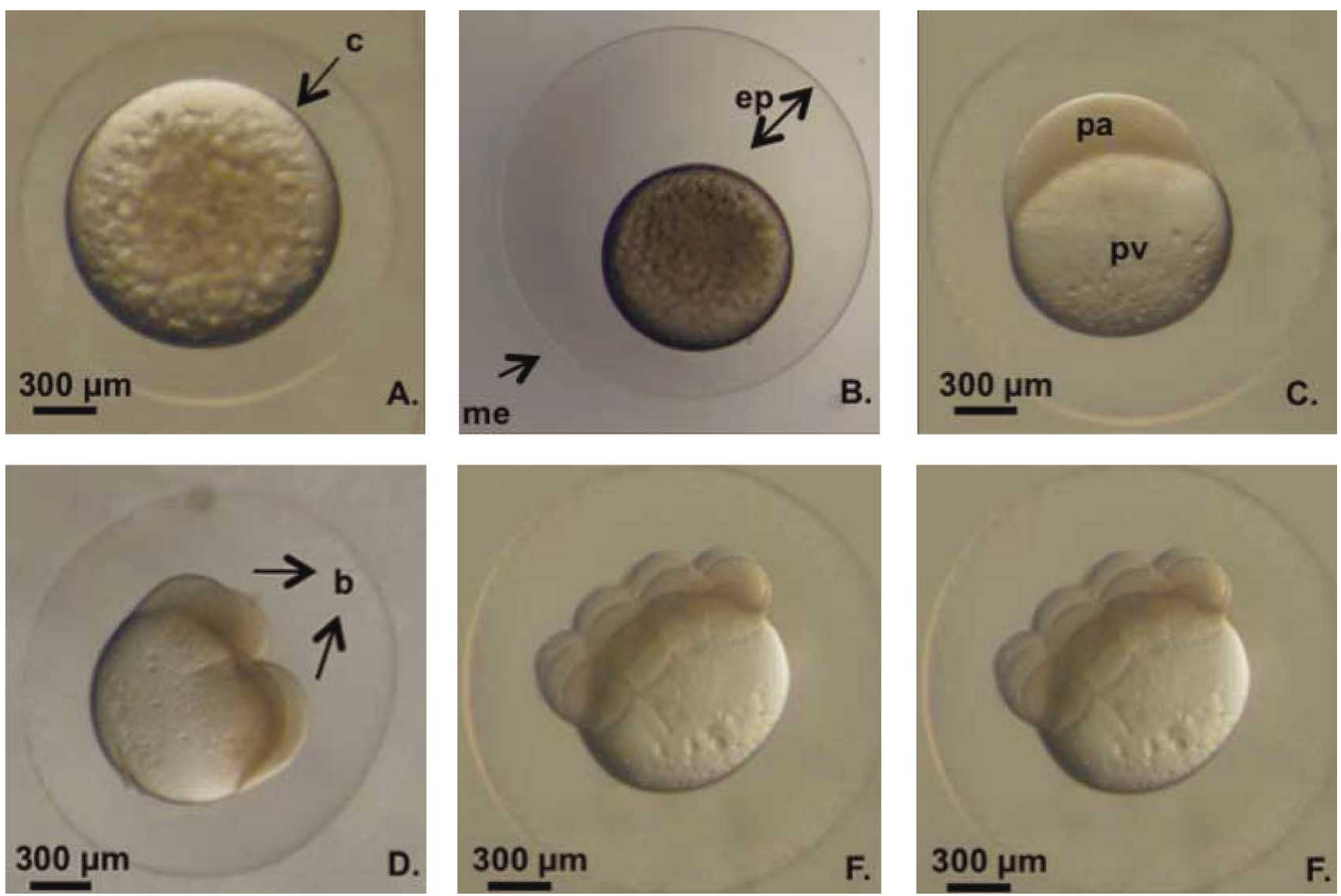

Fig. 1. Blastomeración en el capaz (Pimelodus grosskopfii). A: Oocito recién fertilizado (2x) 0 horas postfertilización (HPF); B: Oocito hidratado (1,5x) 0,15 HPF; C: Blastodisco unicelular (2x) 0,2 HPF; D: 4 blastómeros (2x) 0,45 HPF; E: 8 blastómeros (2x) 0,55 HPF; F: 16 blastómeros (2x) 1 HPF. Corion (c), membrana externa (me), espacio perivitelino (ep), polo animal (pa), polo vegetal (pv), blastómeros (b).

Blastulación. Se observó un complejo de células dispuestas sobre un vitelo no dividido formando el blastodisco; sus células se fusionaron con el vitelo formando el periblasto (1,4 HPF) (Fig. 2 A). A las 1,8 HPF se evidenció la elevación del blastodisco (Fig. 2B), mostrando descenso (2,5 HPF) (Fig. 2C) y posterior achatamiento a las 3,3 HPF, marcando el final de la blastulación y el comienzo de la gastrulación (Fig. 2D).

Gastrulación. A las 3,6 HPF las células blastodérmicas y periblásticas comenzaron a envolver el vitelo mediante movimientos epibólicos (Fig. 3A); posteriormente (4 HPF), el $50 \%$ del vitelo se encontró rodeado por estas células (Fig.
$3 \mathrm{~B})$. A las $5 \mathrm{HPF}$ fue posible diferenciar un eje cráneo caudal (Fig. 3 C), evidenciando el cierre del blastoporo a las 5,7 HPF, cuando la epibolia es del 95\%, lo cual marca el final de la fase de gastrulación (Fig. 3 D).

Organogénesis. En P. grosskopfii se observó la diferenciación cráneo caudal de simetría bilateral y el comienzo de la neurolación, evidenciándose la formación de los primeros somitos (7 HPF) (Fig. 4A); aproximadamente a las 8,5 HPF se observaron las vesículas ópticas, óticas y de Kupffer (Figura $4 \mathrm{~B} \mathrm{y} \mathrm{C)} \mathrm{y} \mathrm{hacia} \mathrm{las} 9 \mathrm{HPF}$ fue posible diferenciar la aleta embrionaria (Figura $4 \mathrm{C}$ ). Posteriormente, a las $10 \mathrm{HPF}$, se evidenció un embrión bien desarrollado con desprendi- 

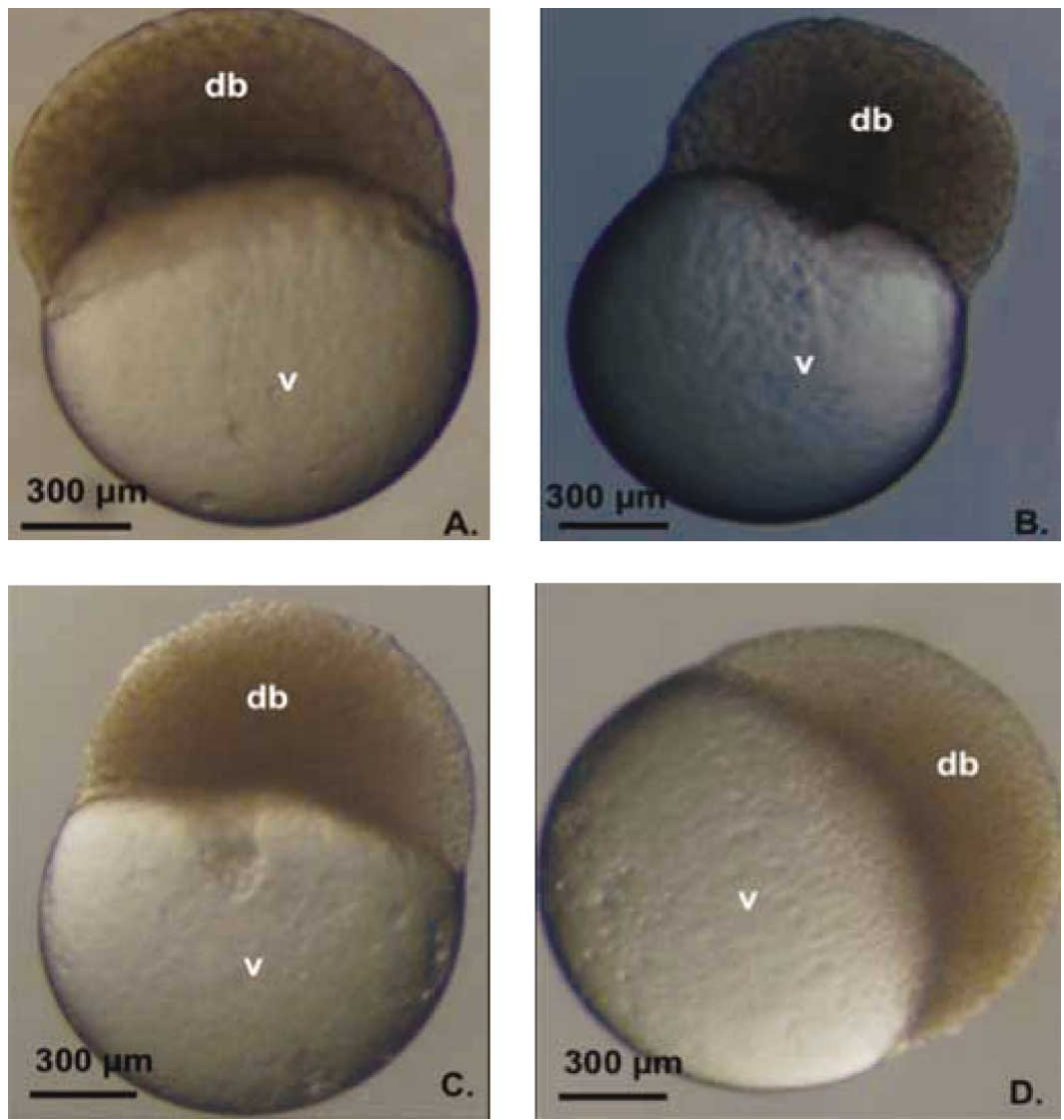

Fig. 2. Blastulación en el capaz (Pimelodus grosskopfii) A: 1,4 HPF; B: 1,8 HPF, C: 2,5 HPF, D: 3,3 HPF. Blastodisco (db), vitelo (v). $(6,4 \mathrm{x})$.
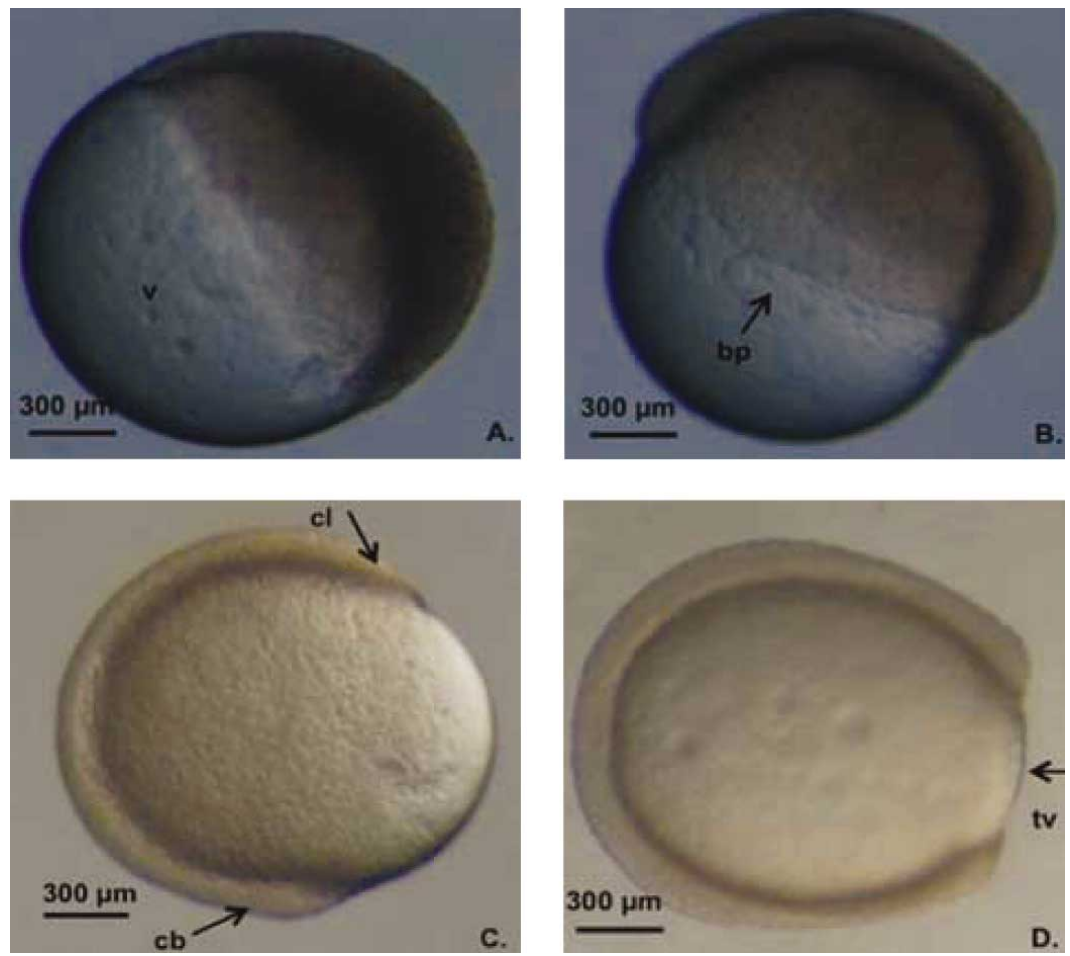

miento de la cola del saco vitelino (Fig. 4D); a las 10,5 HPF se observaron los primeros movimientos autónomos. Transcurridas $11 \mathrm{HP}$, se diferenció el corazón y el inicio de la circulación periférica, con movimientos cada vez más fuertes, acompañados de numerosas contracciones caudales y marcado deterioro de la membrana.

Eclosión. La eclosión se inició a las 11,5 HPF y hacia las 12 HPF más del $90 \%$ de las larvas habían eclosionado. Al eclosionar éstas mostraron ausencia de pigmentación, con una longitud total de $2987 \pm 67 \mu \mathrm{m}$, evidenciándose un tracto digestivo rudimentario sin abertura bucal ni anal, presencia de cromatóforos en el saco vitelino, aleta primigenia bien desarrollada bordeando la región caudal, bulbo olfatorio, corazón, dos otolitos en la vesícula ótica, cápsula óptica y nado errático (Fig. 5).

La Tabla I resume los estadios del desarrollo embrionario del Pimelodus grosskopfii.

\section{DISCUSIÓN}

Los óvulos de peces se clasifican como telolecíticos, ya que presentan una gran cantidad de vitelo de amplia distribución (Ribeiro et al., 1995; Leme dos Santos \& Azoubel, 1996). Los óvulos de $P$. grosskopfii se ajustan a esta clasificación $\mathrm{y}$, por tanto, presentan divisiones meroblásticas o parciales, limitadas al polo animal. Tal división es típica de los huevos de peces (Lagler et al., 1977).

El diámetro promedio de los óvulos en $P$. grosskopfii al momento del desove fue de $1200 \mu \mathrm{m}$ y las larvas presentaron

Fig. 3. Gastrulación en el capaz (Pimelodus grosskopfii) A: 3,6 HPF, B: Epibolia < $50 \%$; 4,0 HPF, C: 5,0 HPF, D: Cierre del blastoporo 5,7 HPF. Vitelo (v), borde del periblasto (bp), cola (cl), cabeza (cb), tapón del vitelo (tv). $(6,4 \mathrm{x})$. 

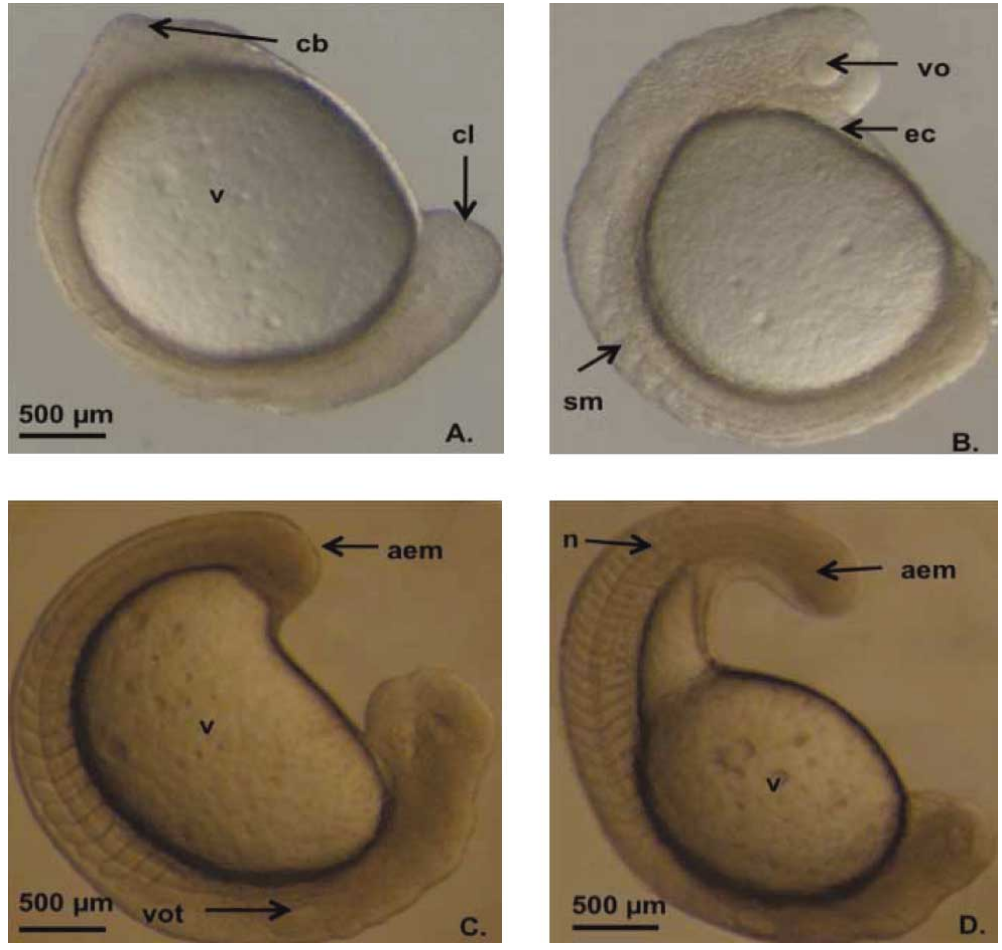

Fig. 4. Organogénesis en el capaz (Pimelodus grosskopfii) A: 7 HPF, B: 8,5HPF, C: 9 HPF, D: 10 HPF. Vitelo (v), cabeza (cb), cola (cl), somitas (sm), vesícula óptica (vo), ectodermo (ec), aleta embrionaria (aem), vesícula ótica (vot), notocorda $(n) .(6,4 x)$.

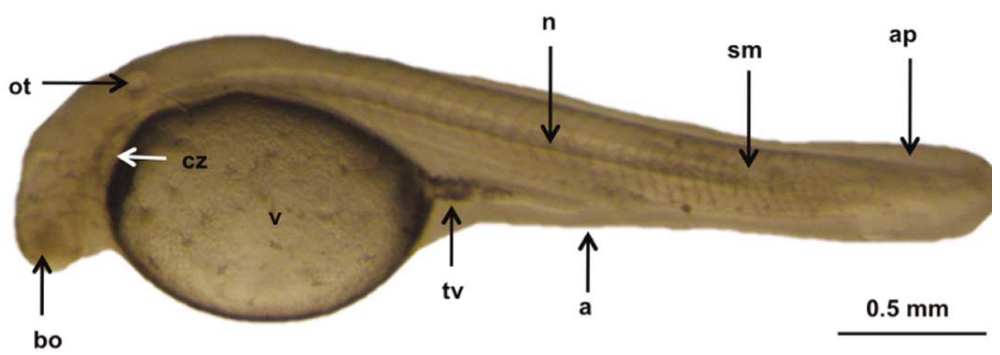

Fig. 5. Larva recién eclosionada de capaz (Pimelodus grosskopfii): bulbo olfatorio (bo), corazón (cz), otolitos (ot), notocorda (n), aleta primigenia (ap), somitas (sm), ano (a), tubo vitelino (tv).

una longitud de $2987 \mu \mathrm{m}$ al momento de la eclosión. Novoa \& CatañoVergara observaron en Sorubim cuspicaudus un diámetro promedio de $1492 \mu \mathrm{m}$ para los óvulos y de $3320 \mu \mathrm{m}$ de longitud de las larvas recién eclosionadas, mientras que Clavijo-Ayala \& Arias-Castellanos (2004a), en Rhamdia sebae, reportaron un diámetro promedio de $930 \mu \mathrm{m}$ para los óvulos. El diámetro de los óvulos es probable que esté relacionado con el modo de fertilización de cada especie, siendo que las especies que presentan fertilización externa, por lo general producen óvulos de menor tamaño que aquellas que poseen fertilización interna (Sánchez et al., 1999).
El diámetro de los óvulos también influye sobre el período de incubación, siendo que óvulos de mayor tamaño tienen un tiempo de incubación más largo que óvulos pequeños (Sargent et al., 1987). El P. grosskopfii produjo óvulos considerados pequeños y presentaron un relativo corto periodo de incubación. La temperatura es otro elemento que juega un papel importante en la incubación. A temperaturas más altas, el tiempo de incubación se acorta, mientras que a temperaturas más bajas, este tiempo se aumenta (Ninhaus- Silveira et al., 2006).

Son pocos los trabajos realizados sobre la ontogenia de silúridos en Colombia; sin embargo, existen reportes para algunas especies de valor comercial. Por ejemplo, Novoa \& Cataño-Vergara observaron que el desarrollo embrionario para Sorubim cuspicaudus, a temperatura de $28,2^{\circ} \mathrm{C}$, ocurría entre 14 - $20 \mathrm{HPF}$, similar a lo encontrado por Zapata-Berruecos et al. (2007) para Pseudoplatystoma sp, en el cual transcurrió durante un periodo de $12-17$ horas a una temperatura de $27^{\circ} \mathrm{C}$, mientras que para capaz ( $P$. grosskopfii) la eclosión se presentó entre las 11,5 - $12 \mathrm{HPF}$ a $27^{\circ} \mathrm{C}$. Estos rangos amplios de duración del desarrollo embrionario que se observan para una misma especie, producen un crecimiento asincrónico de las larvas y por lo tanto heterogeneidad en su tamaño, lo cual favorece el canibalismo entre ellas (Kestemont et al., 2003). La doble membrana ha sido poco estudiada y su función específica aún no ha sido definida, pudiendo ser considerada una característica propia de las especies del orden Siluriformes (Marques et al., 2008). Por otro lado, la presencia de un amplio espacio perivitelino observado en esta especie tiene relación con la incorporación de agua por el óvulo durante la maduración meiótica, fenómeno denominado hidratación llegando a alcanzar el contenido de agua en el huevo y en los embriones tempranos hasta el $95 \%$ de su peso (Cerdà, 2002). Godinho et al., (1978) y Lake (1967) afirmaron que un amplio espacio perivitelino protege al embrión contra las agresiones del ambiente, contribuyendo a su sobrevivencia y confiriéndole flotabilidad positiva en la columna de agua, lo cual le permite obtener mayor aporte de oxígeno, así como mayor dispersión en el medio acuático (Cerdà) . 
VALbUENA, V. R. D.; ZAPATA, B. B. E.; RUALES, C. D. \& CRUZ-CASALLAS, P. E. Desarrollo embrionario del capaz Pimelodus grosskopfii (Steindachner, 1879). Int. J. Morphol., 30(1):150-156, 2012.

Tabla I. Cronología de los principales eventos durante el desarrollo embrionario de capaz (Pimelodus grosskopfii).

\begin{tabular}{lcl}
\hline Estadio & HPF* & Descripción \\
\hline \multirow{2}{*}{ Blastomeración } & 0 & Elevación del corion, formación del espacio perivitelino \\
& 0,2 & Diferenciación del polo animal y vegetal \\
Blastulación & $1,8-1,3$ & Segmentaciones sucesivas del polo animal, formación de blastómeros. \\
Gastrulación & 3,3 & Blastodisco al to y estratificado \\
& 4,0 & Elastodisco achatado \\
& 5,7 & Cierre del blastoporo \\
& 7,0 & Diferenciación cráneo-caudal, inicio de neurolación \\
Organogénesis & 8,5 & Diferenciación de las vesículas ópticas, óticas y vesícula de Kupffer \\
& 10,0 & Liberación de la cola del vitelo. Somitas y saco vitelino bien diferenciados \\
& 10,5 & Primeros movimientos \\
& 11,0 & Diferenciación del corazón e inicio de la circulación periférica, fuertes contracciones \\
& $11,5-12,0$ & Eclosión \\
\hline
\end{tabular}

*HPF $=$ Horas postfertilización.

El patrón de segmentación observado en capaz $(P$. grosskopfii) es de tipo meroblástico; la blastomeración observada para la especie es análoga a la reportada para algunos silúridos como Rhamdia quelen, Pseudoplatystoma corruscans, Leiarius marmoratus (Pereira et al., 2006; Marques et al.; Zapata-Berruecos et al., 2008) y para otras especies del grupo de los carácidos como Brycon amazonicus y Brycon sinuensis (Clavijo-Ayala \& Arias-Castellanos, 2004b; Atencio-García et al., 2006).

Entre los eventos importantes en el desarrollo embrionario de teleósteos se encuentra el cierre del blastoporo, el cual se observó para el capaz ( $P$. grooskopfii) a las 5,7 HPF. Similar situación fue observada para otros silúridos, presentándose en
Pseudoplatystoma sp a las 6 HPF y en Leiarius marmoratus a las 5,3 HPF (Zapata-Berruecos et al., 2007, 2008) y en otras especies reofílicas como Brycon amazonicus a las 6,5 horas a $27,5 \pm 0,2^{\circ} \mathrm{C}$ ( Clavijo-Ayala et al., 2004b) y en Brycon sinuensis a las $6 \mathrm{HPF}$ a $27,8 \pm 0,4^{\circ} \mathrm{C}$ (Atencio-García et al.).

Estos resultados aportan conocimiento a la biología de la especie, proporcionando una base para posteriores estudios en cuanto a la descripción del desempeño en la fase larval y en posteriores trabajos de primera alimentación, siendo útiles para evaluar el efecto de sustancias tóxicas sobre la fauna acuática, como bioindicador de la calidad del ambiente; además de promover estudios taxonómicos, ecológicos y de conservación del P. grosskopfii.

VALBUENA, V. R. D.; ZAPATA, B. B. E.; RUALES, C. D. \& CRUZ-CASALLAS, P. E. Embryonic development of capaz Pimelodus grosskopfii (Steindachner, 1879). Int. J. Morphol., 30(1):150-156, 2012.

SUMMARY: The knowledge of embryonic development in fish is important in native species with potential for fish farming, by virtue of which it makes possible to identify morphological and chronological events to establish management practices during incubation periods and larviculture. The capaz (Pimelodus grosskopfii) is a species with potential for commercial crop, due to their omnivorous eating habits and acceptance of its meat in the market. To study the embryonic development of the species, sexually mature adult specimens were induced to reproduce with carp pituitary extract $\left(5.75\right.$ and $4.0 \mathrm{mg} \cdot \mathrm{Kg}^{-1}$, females and males, respectively). The inseminated oocytes were incubated in an upward flow system 30 a $27 \pm 1^{\circ} \mathrm{C}$. The samples $(\mathrm{n}=30)$ were collected at the same time of the extrusion, during fertilization, and every 15 minutes starting from 0 to 2 hours post fertilization (HPF) and every 30 minutes from 0 to 2 HPF, and every 30 minutes from 2 to 5 HPF; finally, between 5 HPF and hatching every 60 minutes. The fertilized oocytes had a spherical shape without adhesions and large perivitelline space. Embryonic development took $12 \mathrm{HPF}$. The differentiation in animal and vegetal pole occurred at $0.2 \mathrm{HPF}$, the first cleavage at $0.3 \mathrm{HPF}$, stratified and high blastodisc at $1.8 \mathrm{HPF}$, flattened blastodisc at $3.3 \mathrm{HPF}$, the epiboly $<50 \%$ was observed at $4 \mathrm{HPF}$, the closure of the blastopore at 5.7 HPF, cranial-caudal differentiation and starting the neurolation at $7 \mathrm{HPF}$, the differentiation of the optic vesicles, otic and Kupffer's vesicle at $8.5 \mathrm{HPF}$, tail of the vitelum was released at $10 \mathrm{HPF}$, first movements were observed at 10.5 and finally hatching occurred at 12 HPF. When the larvae hatched, they showed a total length of $2987 \pm 67 \mu \mathrm{m}$, without depigmentation, rudimentary digestive system without oral and anal opening and the presence of chromatophores on the yolk sac.

KEY WORDS: Blastomere; Blastulation; Embryonic development; Gastrulation; Organogenesis; Pimelodus grosskopfii. 


\section{REFERENCIAS BIBLIOGRÁFICAS}

Atencio-García, V. J.; Arabia-Ricardo, F. \& Aristizabal-Reginfo, J. Desarrollo embrionario y larvario de Dorada (Brycon sinuensis). IV Congreso Iberoamericano Virtual de Acuicultura, 2006. pp. 604618.

CCI (Corporación Colombia Internacional). Pesca y Acuicultura. Bogotá. INCODER, Ministerio de Agricultura, 2006. pp.138.

Cerdà, J. Mecanismos fisiológicos durante la hidratación del huevo de teleósteos: hacia el desarrollo de nuevos métodos de criopreservación. Bol. Inst. Esp. Oceanogr.,18:145-52, 2002.

Clavijo-Ayala, J. \& Arias-Castellanos, J. A. Desarrollo embrionario de Rhamdia sebae c.f. II Congreso Colombiano de Acuicultura. X Jornada de Acuicultura IALL, Universidad de los Llanos, Villavicencio, Colombia, 2004a. pp.41.

Clavijo-Ayala, J. A. \& Arias-Castellanos, J. A. Avances en el estudio de la embriología del Yamú (Brycon Siebenthalae) (Pisces: Characidae). Dahlia. Rev. Asoc. Colomb. Ictiol., 7:37-48, 2004b.

Godinho, H.; Fenerich, N. \& Narahara, M. Desenvolvimento embrionário e larval de Rhamdia hilarii (Valencienses, 1840) (Siluriformes Pimelodidae). Rev. Bras. Biol., 38:151-6, 1978.

Johnston, I. I.; Vera, V. V. \& Abercromby, M. Temperature and myogenesis in embryos of the Atlantic herring Clupea harengus. $J$. Exp. Biol., 198:1389-403, 1995.

Kestemont, P.; Jourdan, S.; Houbart, C. C.; Paspatis, M.; Fontaine, P.; Cuvier, A.; Kentouri, M. \& Baras, E. Size heterogeneity, cannibalism and competition in cultured predatory fish larvae: biotic and abiotic influences. Aquaculture, 227:333-56, 2003.

Lagler, K. F.; Bardach, J. E.; Miller, R. R. \& Passino, D. R. M. Ichthyology. New York, John Wiley \& Sons, 1977. pp.528.

Lake, J. Rearing experiments with five species of Australian freshwater fishes. I. Inducement to spawning. Aust. J. Mar. Freshwat. Res., 18:137-54, 1967.

Leme dos Santos, H. S. \& Azoubel, R. Embriologia comparada: Texto y Atlas. São Paulo, FUNEP, Jaboticabal, 1996. pp.189.

Marques, C.; Okada-Nakaghi, L. S.; Faustino, F.; Nakaghi-Ganeco, L. \& Senhorini, J. A. Observation of the embryonic development in Pseudoplatystoma corruscans (Siluriformes: Pimelodidae) under light and scanning electron microscopy. Zygote, 16:333-42, 2008.

Mojica, J. I. Lista preliminar de las especies dulceacuícolas de Colombia. Rev. Acad. Colomb. Cienc. Exactas, Fis. Nat., 23:547-66, 1999.

Ninhaus-Silveira, A.; Foresti, F. \& de Azevedo, A. Structural and ultrastructural analysis of embryonic development of Prochilodus lineatus (Valenciennes, 1836) (Characiforme; Prochilodontidae). Zygote, 14:217-29, 2006.

Novoa, J. D. \& Cataño-Vergara, Y. M. Descripción del desarrollo em- brionario y larvario del blanquillo (Sorubim cuspicaudus). Trabajo de Grado Profesional en Acuicultura. Montería, Colombia, Facultad de Medicina Veterinaria y Zootecnia, Universidad de Córdoba, 2005. pp.102.

Ortega-Lara, A.; Murillo, O.; Pimienta, C. \& Sterling, E. Los peces del Alto Cauca, riqueza ictiológica del Valle del Cauca. Santiago de Cali, Colombia, Corporación Autónoma Regional del Valle del Cauca-CVC, 2000. pp.69.

Pereira, C. R.; Barcellos, L. J.; Kreutz, L. C.; Quevedo, R. M.; Ritter, F. \& Silva, L. B. Embryonic and Larval Development of Jundiá (Rhamdia quelen, Quoy \& Gaimard, 1824, Pisces, Teleostei) a South American Catfish. Braz. J. Biol., 66:1057-63, 2006.

Ribeiro, C. R.; Leme dos Santos, H. S. \& Bolzan, A. A. Estudo comparativo da embriogênese de peixes ósseos (Pacu, Piaractus mesopotamicus, Tambaqui, Colossoma macropomum e híbrido Tambacú). Rev. Bras. Biol., 55:65-78,1995.

Sánchez, P. V.; Nakatani, K. \& Bialetzki, A. Morphological description of the developmental stages of Parauchenipterus galeatus (Linnaeus, 1766) (Silurifores, Auchenipteridae) on the floodplain of the upper Paraná River. Rev. Bras. Biol., 59:429-38, 1999.

Sargent, R. C.; Taylor, P. D. \& Gross, M. R. Parental care and evolution of egg size in fishes. Am. Nat., 129:32-46, 1987.

Villa-Navarro, F. A. Diferenciación entre poblaciones de Pimelodus clarias y Pimelodus grosskopfii (Siluriformes: Pimelodidae) en la cuenca del río Magdalena (Colombia). Tesis de Maestría, Cali, Colombia, Facultad de Ciencias, Universidad del Valle, 2002. pp. 100 .

Villa-Navarro, F. A. Estudio biológico pesquero de la represa de Prado para la determinación de especies promisorias en acuicultura. Ibagué, Colombia, Universidad del Tolima. 1999. pp.105.

Zapata-Berruecos, B. E.; Medina-Robles, V. M.; Mira-López, T. M.; Otero-Paternina, A. M.; Ramírez-Merlano, J. A.; VelascoSantamaría, Y. M. \& Cruz-Casallas, P. E. Descripción preliminar del desarrollo embrionario de bagre rayado (Pseudoplatystoma fasciatum). Villavicencio, Colombia, XIII Jornada de Acuicultura Universidad de los Llanos, 2007. pp.60-4.

Zapata-Berruecos, B. E.; Ramírez-Merlano, J. A.; Otero-Paternina, A. M.; Medina-Robles, V. M.; Velasco-Santamaría, Y. M. \& CruzCasallas, P. E. Descripción preliminar del desarrollo embrionario de yaque Leiarius marmoratus. IV Congreso Colombiano de Acuicultura. Rev. Colomb. Cienc. Pecu., 21:513, 2008.

\section{Dirección para correspondencia:}

Pablo E Cruz-Casallas

Grupo de Investigación sobre Reproducción y Toxicología de Organismos Acuáticos - GRITOX. Instituto de Acuicultura Facultad de Ciencias Agropecuarias y Recursos Naturales Universidad de los Llanos

Villavicencio

Recibido : 12-02-2011

COLOMBIA

Aceptado: 30-12-2011

Email: pecruzcasallas@unillanos.edu.co 